\title{
The protection of LingqiHuangban Granule on human retinal endothelial cells against oxidative stress-induced injury by network pharmacology
}

\section{Zhenzhen Zhang}

Department of Ophthalmology, Affiliated Ninth People's Hospital of Shanghai, Shanghai Jiaotong University school of medicine, Shanghai, China

\section{Chuandi Zhou}

Department of Ophthalmology, Affiliated Shanghai General Hospital, Shanghai Jiaotong University, National Clinical Research Center for Eye Diseases, Shanghai Key Laboratory of Ocular Fundus Diseases, Shanghai Engineering Center for Visual Science and Photo

\section{Deji Draga}

Department of Ophthalmology, Shigatse People's Hospital, Shigatse, Xizang, China

\section{Ihamo Thashi}

Department of Ophthalmology, Shigatse People's Hospital, Shigatse, Xizang, China

\section{Zhi Zheng}

Department of Ophthalmology, Affiliated Shanghai General Hospital, Shanghai Jiaotong University, National Clinical Research Center for Eye Diseases, Shanghai Key Laboratory of Ocular Fundus Diseases, Shanghai Engineering Center for Visual Science and Photo

\section{Qinghua Qiu ( $\nabla$ qinghuaqiu@163.com )}

Shanghai Jiao Tong University School of Medicine

\section{Research}

Keywords: Chinese herbal medicine, Human retinal endothelial cells (HRECs), LingqiHuangban Granule(LQHBG), Oxidative stress

Posted Date: May 20th, 2020

DOI: https://doi.org/10.21203/rs.3.rs-28861/v1

License: (c) (i) This work is licensed under a Creative Commons Attribution 4.0 International License. Read Full License 


\section{Abstract}

Background: LingqiHuangban Granule(LQHBG) is a famous traditional Chinese medicine formula used to manage retinal diseases, as an effective holistic treatment through warming Yang to exert tonifying effects on kidney and invigorating spleen to remove dampness to nourish essence of effect. The study examined protection of LQHBG on oxidative stress-induced injury in human retinal endothelial cells(HRECs) in vitro, determined the potential molecular targets of LQHBG using network pharmacology.

Methods: The potential targets of active ingredients in LQHBG were predicted using pharmmapper. Gene Ontology (GO) and Kyoto Encyclopedia of Genes and Genomes(KEGG) pathway enrichment analyses were carried out using Molecule Annotation System. The protein-protein interaction networks were constructed using Cytoscape. LQHBG was administered to rabbits to prepare medicated serum. The apoptosis of HRECs was evaluated by TUNEL and Flow Cytometry(FCM). MDA, SOD, LDH, GSH-Px, and TAOC were detected. The mRNA expressions of Nrf2, NF-KB and HO-1 were detected, protein expression levels of Nrf2, Bcl-2, NF-KB, HO-1 and caspase-3 were analyzed.

Results: TUNEL demonstrated the numbers of apoptotic cells in low-and high-dose LQHBG groups was obviously less than model group $(\mathrm{P}<0.05)$. FCM analysis revealed apoptotic rates of HRECs in low-and high-dose LQHBG groups were obviously reduced in a dose-dependent manner $(P<0.05)$. The potential mechanism of LQHBG was the NF-KB pathway identified using PharmMapper. LQHBG significantly decreased MDA, LDH levels and enhanced SOD, GSH-Px and T-AOC generation. LQHBG inhibited upregulation of NF-KB, caspase-3 and enhanced Bcl-2, Nrf2, and HO-1 expression.

Conclusion: LQHBG protected HRECs against oxidative-stress via suppression of apoptosis and elevation of antioxidant ability, which may involve activation of Nrf2/ARE/HO-1 pathway and inhibition of NF-KB pathway.

\section{Background}

Ischemia-related retinal diseases include age-related macular degeneration(AMD), central retinal artery occlusion(CRAO), central retinal vein occlusion(CRVO), branch retinal artery occlusion(BRAO), branch retinal vein occlusion(BRVO), and glaucoma, which result in serious complications(Osborne et al., 2004, Levin and Louhab., 1996). Therefore, the treatment of retinal ischemia is important to patient outcomes. Ischemia-induced retinal disorders increase oxidative stress, and one direct target is human retinal endothelial cells(HRECs)(Masuda, Shimazawa and Hara., 2017, Castilho et al., 2012).

Endothelial dysfunction is a primary feature of ischemia retinopathies, which originate with endothelial cell damage, followed by extensive capillary regression that ultimately leads to widespread inner retinal ischemia and hypoxia(Leal et al., 2009, Shi et al., 2015, Zhang et al., 2014). Hypoxia induces capillary endothelial cell apoptosis and results in retinal capillary obliteration, which deregulates the growth of newblood vessels that protrude from retinal surface and aggravate retinal ischemia injury(Deliyanti et al., 
2018). Chronic oxidative stress causes HREC dysfunction and apoptosis, which lead to the deterioration of eye diseases(Atienzar Aroca et al., 2016).

LQHBG, a famous traditional Chinese medicine formula that is used to manage retinal diseases, such as AMD, CRAO, CRVO, BRAO, and BRVO(Li et al., 2012, Gong et al., 2008), consisting of 10 crude herbs: Cistanche( RouCongRong), Lyciumbarbaruml(GouQiZi), Semen Cuscutae(TuSiZi), Codonopsispilosula(DangShen), Ganoderma(LingZhi), Rhizomaatractylodis(CangZhu), Salvia miltiorrhiza(DanShen), Angelica aculeolata(DangGui), Ligusticumwallichii(ChuanXiong), Sargassum(HaiZao) in a 3:2:2:2:2:2:2:2:2:2:2 ratio, which was developed by the First People's Hospital affiliated with Shanghai Jiaotong University(Shanghai medicine system word: Z05050795). XingWei Wu et al. suggested that LQHBG in combination with intravitreal ranibizumab injection effectively stabilizes and improves vision with fewer intravitreal injections, which may be an interesting option in this type of patients(Lu et al., 2016). Yuanyuan Gong et al. also indicated that LQHBG protects retinal function and morphology from light-induced retinal damage in rats(Gong et al., 2008).

Network pharmacology is a distinctive new approach based on advances in network biology and polypharmacology, providing a more comprehensive understanding of the targets, mechanism, and pathways behind drug action. PharmMapper provides deeper insights and scientific evidence for TCM and helps identify potential targets of chinese herbs and their underlying mechanisms (Tang and Aittokallio., 2014; Poornima et al., 2016). The Encyclopedia of Traditional Chinese Medicine (ETCM, http://www.nrc.ac.cn:9090/ETCM/) (Xu et al., 2018) was used to identify the candidate target genes of each herb contained in LQHBG. In the present study, investigations based on the pharmacology database, potential signaling pathway were predicted using the network pharmacology method. The current research investigated the protective effects of LQHBG in HRECs under hydrogen peroxide-induced oxidative stress injury and used the serum pharmacology method for drug administration. TUNEL and FCM assays were used to measure apoptosis of HRECs, and MDA, SOD, LDH, GSH-Px, and T-AOC analyses were used to measure oxidative stress levels. We also investigated the underlying mechanisms. The expressions of Nrf2, NF-KB and HO-1mRNAs were identified by PCR, and protein expressions of Nrf2, $\mathrm{Bcl}-2$, NF-kB, HO-1 and caspase-3 were analyzed by Western blot.

\section{Methods}

\section{Pharmacological Network construction}

The Encyclopedia of Traditional Chinese Medicine (ETCM, http://www.nrc.ac.cn:9090/ETCM/) (Xu et al., 2018) was used to identify the candidate target genes of each herb contained in LQHBG. The network was constructed using Cytoscape 3.7.1(Shannon et al., 2013) with the protein-protein interaction (PPI) data from High-quality INTeractomes (HINT; version 4, http://hint.yulab.org)(Das J et al., 2012), and major genes were screened after the calculation of topological features. Kyoto Encyclopedia of Genes and Genomes (KEGG) pathway enrichment analysis of major genes was conducted using the Database for Annotation, Visualization and Integrated Discovery (DAVID; version 6.8, https://david.ncifcrf.gov/) 
(Huang Da W et al., 2009). The herb-gene-pathway network was constructed using Cytoscape 3.7.1 to visualize the relationships among herbs, genes, and pathways.

\section{Preparation of LQHBG}

LQHBG consists of ten herbs and the mixed proportion of respective herb in LQHBG is illustrated in Table1. All the mentioned herbal names can be checked in accordance with the database of www.theplantlist.org. LQHBG was developed by the First People's Hospital Affiliated with Shanghai Jiaotong University(Shanghai medicine system word: Z05050795).

\section{Reagents}

DMEM, fetal bovine serum and trypsin were purchased from GIBCO company (United States).Two dimethyl sulfoxide sulfone (dimethylsulfoxide, DMSO) were purchased from Sigma. An annexin $\mathrm{V} /$ propidium iodide $(\mathrm{PI})$ apoptosis detection kit was purchased from R\&D company. A terminaldeoxynucleotidyl transferase-mediated deoxyuridine triphosphate-biotinnick end labeling(TUNEL) kit was purchased from America Promega. A Western blotting kit (AC031, AB126, antibody) was purchased from Beyotime Company.

\section{Main instruments}

A constant-temperature carbon dioxide cell incubator (MCO-20AIC, Sanyo Company), refrigerated centrifuge (Centrifuge5418R, Eppendorf Company), inverted phase contrast microscope (BX41-PHD, PII, Olympus Company), fluorescence microscope (AxioVertS100, Zeiss Company), flow cytometry (GuavaeasyCyte8HT, Millipore Company) instrument, and confocal laser scanning microscopy (LSM510Meta, Zeiss Company) were used.

\section{HPLC analysis}

LQHBG were analyzed by Agilent HPLC instrument(Agilent, USA) equipped with a Eclipse XDB$\mathrm{C}_{18}(250 \mathrm{~mm} \times 4.6 \mathrm{~mm} 5, \mu \mathrm{m})$ column and an evaporative light scattering detector(ELSD). The drift tube temperature of the ELSD system was set to $30^{\circ} \mathrm{C}$ and the auxiliary gas flow rate was $1.0 \mathrm{~L} / \mathrm{min}$. The mobile phase consisted of acetonitrile $(A)$ and water $(B)$ at a flow rate of $1.0 \mathrm{~L} / \mathrm{min}$. A gradient program was used as follows: 0-8min:A-B(20:80), 8-18min: from A-B(20:80) to A-B(30:70)凶18-20min: A$B(30: 70), 20-23$ min: from $A-B(30: 70)$ to $A-B(20: 80)$. The detection wavelength was set at $334 \mathrm{~nm}$ and flow rat was maintained at $1.0 \mathrm{ml} / \mathrm{min}$. The typical HPLC-ELSD chromatogram of LQHBG was shown in Fig.2, and the contents of acteoside and salvianolic acid in LQHBG were $36.9 \mathrm{ug} / \mathrm{g}$ and $344 \mathrm{ug} / \mathrm{g}$, respectively.

\section{Serum containing drug}

LQHBG (approval number for the Shanghai pharmacists Z05050795) was purchased from the First Affiliated Hospital of Shanghai Jiao Tong University Department of Pharmacy, Shanghai, PR China. It consists of 10 components: Cistanche(RouCongRong), Lyciumbarbaruml( GouQiZi), Semen 
Cuscutae(TuSiZi), Codonopsispilosula(DangShen), Ganoderma(LingZhi), Rhizomaatractylodis(CangZhu), Salvia miltiorrhiza(DanShen), Angelica aculeolata(DangGui), Ligusticumwallichii(ChuanXiong), Sargassum(HaiZao). Male chinchilla rabbits, body mass $1.2-1.5 \mathrm{~kg}$, were provided by the Shanghai Experimental Animal Center of Chinese Academy of Sciences (Permit No.SCXK(Shanghai) 2007-0005). Animals received humane care in accordance with the Association for Research in Vision and Ophthalmology(ARVO) Statement for the Use of Animals in Ophthalmic and Vision Research. Twelve male chinchilla rabbits, body mass $(2.0 \pm 0.2) \mathrm{kg}$, were randomly divided into a traditional Chinese medicine group $(n=8)$ and control group $(n=4)$. The traditional Chinese medicine, low-dose and high-dose groups( $\mathrm{n}=4$ rats in each group) received a clinical adult dose (according to the average body weight of $60 \mathrm{~kg}), 10$ times and 20 times the daily dose, the daily dose of a $30 \mathrm{~g} / 60 \mathrm{~kg} \times$ rabbit body weight $(\mathrm{kg}) \times 10$ times or 20 times, divided into 8:00am and 8:00pm administration, for 3 consecutive days. During the periods of feeding and fasting (12h), abdominal blood was collected every $1 \sim 2 \mathrm{~h}$ under sterile conditions with an injection of $1 \%$ pentobarbital sodium ( Sigma, USA), the control group was given the same amount of normal saline, and the blood was collected at the same time for 3 days, and then the rabbits were sacrificed. Blood was stored at $4^{\circ} \mathrm{C}$ in the refrigerator overnight and centrifuged at $3000 \mathrm{r} / \mathrm{min}$ (centrifugal radius $10 \mathrm{~cm}$ ) for $25 \mathrm{~min}$. The supernatant was subjected to $0.22 \mu \mathrm{m}$ filter sterilization. Filtered samples from both groups were heated to $56^{\circ} \mathrm{C}$ for $30 \mathrm{~min}$ and stored at $-20^{\circ} \mathrm{C}$.

\section{Cell culture}

HRECs were purchased from Sciencell(Research Laboratories, Inc., Carlsbad, CA, USA). The cell were cultured in human endothelial medium with $5 \%$ fetal bovine serum and $1 \%$ endothelial cell growth supplement at $37^{\circ} \mathrm{C}$ with $5 \% \mathrm{CO}_{2}$ and $95 \%$ ambient air. HRECs were inoculated into 5 large dishes at $2 \star 105 / \mathrm{mL}$. Disposable culture fluid and PBS were added to the following groups: control group serum, serum-free DMEM/F culture liquid plus $\mathrm{H}_{2} \mathrm{O}_{2}(500 \mu \mathrm{mol} / \mathrm{L})$, control group serum and $\mathrm{H}_{2} \mathrm{O}_{2}(500 \mu \mathrm{mol} / \mathrm{L})$, low-dose drug serum plus $\mathrm{H}_{2} \mathrm{O}_{2}(500 \mu \mathrm{mol} / \mathrm{L})$, high-dose drug serum plus $\mathrm{H}_{2} \mathrm{O}_{2}(500 \mu \mathrm{mol} / \mathrm{L})$. Cells were cultured for $24 \mathrm{~h}$, digested and collected with $0.25 \%$ trypsin.

\section{Flow cytometry}

Cell apoptosis was analyzed with FCM analysis. After treatment, cells were harvested and washed twice with PBS, resuspended in 500 $\mu \mathrm{L}$ binding buffer, then stained with Annexin V-FITC and propidium iodide $(\mathrm{PI})$, followed by incubation at room temperature for $15 \mathrm{~min}$ in the dark. After washing with binding buffer, the cells were analyzed by FCM. The above experiments were repeated 3 times.

\section{TUNEL detection}

HRECs were inoculated to preset coverslips in 24-well plates at a density of $5 * 10^{4} / \mathrm{mL}$. Two wells were used for each treatment group. Cells were grown to a monolayer, the culture fluid was discarded, and cells 
were washed with PBS. Cells were cultured for $24 \mathrm{~h}$, and the coverslips were removed. Apoptotic cell detection was performed according to the TUNEL kit.

\section{Total RNA extraction and quantitative real-time PCR}

Retinas were harvested ( $\mathrm{n}=6$ per group) $24 \mathrm{~h}$ after ischemia. Total RNA was extracted from tissues using TRIzol reagent (Invitrogen) and reverse transcribed using M-MLV Reverse Transcriptase. Reversetranscribed cDNA in triplicate samples was used to detect target mRNA level using quantitative real-time PCR and Power SYBR Green PCR Master Mix (Applied Biosystems) in an ABI Prism 7500 sequence detector (Applied Biosystems). The following primers were used: H0-1 forward (5'AGGAGCCTATGGCATCTTC-3') and reverse (5'-GCCGTGTCAACAAGGATAC-3') primers; Nrf2 forward (5'-CAAGTCCAGAAGCCAAAC-3') and reverse (5'-GATGCTGCTGAAGGAATC-3') primers; NF-kB forward (5' GAATGGCTCGTCTGTAGTG-3') and reverse (5'-TGGTATCTGTGCTCCTCTC-3') primers.GAPDH was used as a control. The $10 \mathrm{ml}$ samples contained $1 \mu$ l Power SYBR Green PCR Master Mix (Applied Biosystems), $5 p m o l$ of each primer, and $0.25 \mu$ of the RT reaction. Experiments were performed in triplicate in optically clear 96 well plates (Corning, New York, USA). The cycling parameters consisted of $95^{\circ} \mathrm{C}$ for $10 \mathrm{~min}$, followed by 40 cycles of $95^{\circ} \mathrm{C}$ for $15 \mathrm{~s}, 60^{\circ} \mathrm{C}$ for $1 \mathrm{~min}$, and $72^{\circ} \mathrm{C}$ for $30 \mathrm{~s}$. The relative levels of target mRNA expression were calculated using the $2^{-\Delta \Delta \mathrm{Ct}}$ method.

\section{Protein expression of caspase-3, Bcl2, Nrf2, HO-1 and NF-kB}

HRECs at $5 \times 10^{5} / \mathrm{mL}$ were inoculated in a large dish with $10 \%$ fetal bovine serum $D M E M / F_{12}$ culture solution (1:1). Cells were incubated ay $37^{\circ} \mathrm{C}$ in a cell culture box with $5 \% \mathrm{CO}_{2}$ for $72 \mathrm{~h}$. The cell density was $80-90 \%$. The supernatant was discarded, and cells were rinses 2 times in PBS. Cells were assigned to the 5 groups described above and incubated at $37{ }^{\circ} \mathrm{C}$ in $5 \% \mathrm{CO}_{2}$ for $24 \mathrm{~h}$. The supernatant was discarded, and cells were rinsed with cold PBS 3 times. Lysis buffer was added for several minutes, and cells were scratched. The lysate and cell debris were co-transferred to centrifugation tubes and centrifuged at $4{ }^{\circ} \mathrm{C}$ at 12000r/min. Protein expression was detected using Western blotting (anti-caspase3 antibody, 1:1000, Cell Signaling Technology; anti-NF-KB antibody, 1:1000, Abcam; anti-HO-1 antibody $\otimes 1: 1000$, Abcam; antiNrf2 antibody, 1:1000; Stressgen Biotechnologies; anti-Bcl2 antibody, 1:1000, Abcam; anti- $\beta$ actin, 1:2000; Santa Cruz Biotechnology). These experiments were repeated 3 times.

\section{MDA level and SOD, GSH-PX, CAT and T-AOC activity}

Analysis kits for MDA, SOD, GSH, CAT, and T-AOC were purchased from Beyotime Biotechnology (Jiangsu, China). The GSH-PX, CAT, SOD, MDA, and T-AOC contents were measured using assay kits in strict accordance with the manufacturers' instructions. MDA contents are expressed as $\mathrm{nmol} / \mathrm{ml}$ protein. SOD contentsare expressed as $\mathrm{U} / \mathrm{ml}$ protein, $\mathrm{LDH}$ contents are expressed as $\mathrm{U} / \mathrm{L}$ protein, GSH-PX are expressed as $\mathrm{U} / \mathrm{g}$ protein, T-AOC contents are expressed as $\mathrm{g} / \mathrm{L}$ protein. 


\section{Statistical analysis}

Data analysis was performed using SAS6.12. Data were measured using the mean \pm SD. Multiple sets of data between the single factor analysis of variance. Student's $t$-test was used for comparisons between two groups. $P<0.05$ was statistically significant.

\section{Results}

\section{Pharmacological Network construction}

We identified 1439 candidate target genes of LQHBG, including 1157 of GouQiZi, 1066 of DanShen, 1043 of DangGui, 1027 of ChuanXiong, 1438 of TuSiZi, 1364 of RouCongRong, 1050 of DangShen, 964 of CangZhu, 1214 of LingZhi, 1166 of HaiZao (Table S1). We identified 125 major genes (Degree $\geq 6$ ) after the calculation of topological features (Table S2), and analyzed 114 KEGG pathways using DAVID (Table S3). We selected 6 interesting pathways for network construction (Figure 1): TNF signaling pathway, HIF1 signaling pathway, VEGF signaling pathway, NF-kappa B signaling pathway.

\section{Flow cytometry}

The normal control group exhibited the fewest number of apoptotic cells, and the apoptosis rate was $1.9 \pm 0.16 \%$ (Figure3A). Serum levels of cell apoptosis were similar in model group and model plus serum group $(12.70 \pm 1.37 \%$ and $12.5 \pm 0.94 \%$, respectively) (Figure3B, C). The difference between the two groups was not significant $(P>0.05)$. Apoptosis was significantly reduced in low-dose drug plus serum group $(10.8 \pm 0.55 \%)$ and high-dose drug plus serum group(8.50 \pm 0.39$)$ (Figure3D, E). These two groups were significantly different $(P<0.01)$ from model group. However, the difference between the two groups was also significant $(P<0.05)$ (Figure3F).

\section{TUNEL staining}

TUNEL staining was negative in normal control group, and no yellow HREC nuclei were observed (Figure 4A). Visible large yellow TUNEL-positive staining was observed in HREC nuclei in model group and model serum group(Figure 4B, C). Small yellow TUNEL-positive staining of HREC nuclei were detected in lowdose drug plus serum group (Figure 4D). Yellow TUNEL-positive staining in nuclei of HREC was further reduced in high-dose drug plus serum group, which was significantly less than that in model group (Figure 4E). The difference between the low-dose and high-dose drug plus serum groups was significant $(P<0.05)$ (Figure4F).

\section{LQHBG suppresses $\mathrm{H}_{2} \mathrm{O}_{2}$-induced mRNA expression of $\mathrm{HO}-1$,NF-KB and $\mathrm{Nrf} 2$}

The qRT-PCR analysis examined the mRNA expression of NF-KB, Nrf2, and HO-1 in HRECs, and $\mathrm{H}_{2} \mathrm{O}_{2}$ significantly enhanced NF-KB transcription. LQHBG dose-dependently inhibited NF-KB gene expression (Figure5B). $\mathrm{H}_{2} \mathrm{O}_{2}$-induced oxidative stress increased the expression of $\mathrm{Nrf2}$ and $\mathrm{HO}-1$ genes. LQHBG 
partially reversed this increase, and dose-dependently attenuated Nrf2 and HO-1 gene expression(Figures $5 A, C)$.

\section{Protein expression of caspase-3, Bcl-2, NF-KB, Nrf2, and HO-1}

Protein expression was measures at the relative intensity of gray value (Figure $6 \mathrm{~A}$ ). Caspase-3 and NF-KB protein were expressed at low levels in HRECs of normal control group. Caspase-3 and NF-KB protein expressions were higher in model and model serum groups than in normal control group. Caspase-3 and NF-KB protein expressions were lower in low-dose drug plus serum group than in model group. The highdose drug serum group expressed lower than low-dose drug plus serum group(Figure 6D,E) $(P<0.05)$. $\mathrm{Nrf2}, \mathrm{Bcl} 2$ and $\mathrm{HO}-1$ proteins were detected in normal control group. The levels of these proteins were significantly lower in model and model serum groups than normal control group. LQHBG induced a dosedependent increase in $\mathrm{Bcl} 2, \mathrm{Nrf} 2$ and $\mathrm{HO}-1$ protein expression compared to model and model serum groups $(P<0.05)$ (Figure 6A,B,C,F).

\section{MDA and LDH levels and SOD, GSH-Px and T-AOC activities}

The MDA and LDH levels in model and model plus serum groups were significantly higher than normal control group(Figure 7) $(P<0.05)$. LQHBG treatment decreased these levels in a dose-dependent manner $(p<0.05)$ (Figure 7B,C). SOD, GSH-Px, and T-AOC activities were significantly lower in model and model plus serum groups compared to normal control group. The activities of these enzymes were dosedependently higher in LQHBG-treated groups than the model group $(p<0.05)$ (Figure 7A,D,E).

\section{Discussion}

Ischemia is an important pathological mechanism in retinal diseases. Most cell death associated with retinal ischemia is due to programmed cell death (i.e., apoptosis)(Levin and Louhab., 1996, Zhang et al., 2005). The retina is highly dependent on the oxygen supply, and ischemia-induced oxidative stress is a direct cause of retinal damage(Bonne, Muller and Villain., 1998). Reactive oxygen species(ROS) are a variety of molecules and free radicals derived from molecular oxygen, including superoxide, hydroxyl radicals, hydrogen peroxide $\left(\mathrm{H}_{2} \mathrm{O}_{2}\right)$, and singlet oxygen(Di Mascio, Murphy and Sies., 1991). These molecules are produced during the ischemia-reperfusion process. Free radicals lead to the destruction of DNA molecules and result in energy exhaustion and cell death(Erdelyi et al., 2005). Endothelial-sourced ROS are important physiologically and pathologically in many ischemic retinopathies(PellegriniGiampietro et al., 1990) .

MDA and LDH are biomarkers of oxidative stress levels(Anderson, Maude and Nielsen., 1985), and these proteins are often used to evaluate oxidative stress-induced endothelial injury(Chen et al., 2009). Enzymatic antioxidant enzymes, such as SOD, GSH-Px, and T-AOC, play key roles in neutralizing the ROS that are produced during stress conditions, and these enzymes are essential in defending against endothelial damage(Agardh et al., 2006, Lewden et al., 1996). Accumulated ROS during antioxidant responses in endothelial cells, and maintaining a balance is essential for homoeostasis in endothelial 
cells(Shi et al., 2015). Our results demonstrated that LQHBG significantly inhibited the formation of intracellular ROS in $\mathrm{H}_{2} \mathrm{O}_{2}$-stimulated HRECs. LQHBG notably decreased MDA and LDH levels and increased SOD, GSH-Px, and T-AOC activity levels. These results suggest that the protective effect of LQHBG is associated with the inhibition of intracellular ROS production.

The present study used the serum pharmacological method to investigate the protective effect of LQHBG on oxidative stress in vitro. FCM and TUNEL tests confirmed that LQHBG-medicated serum inhibited HREC apoptosis under oxidative stress. Further studies on apoptotic signal transduction molecules revealed that LQHBG played a protective role via the downregulation of NF-KB, caspase-3 and upregulation of $\mathrm{Bcl}-2$ to inhibit hypoxia-induced HREC apoptosis, which was consistent with the analysis of network pharmacology.

Nuclear factor (erythroid-derived 2)-like 2 (Nrf2) is a critical transcription factor that regulates the antioxidant defense system(de Vries et al., 2008, Enomoto et al., 2001, Alfieri et al., 2011). Nrf2 controls the antioxidant response elements (AREs) located in the promoter regions of its target genes and activates downstream gene expression, including the antioxidant enzymes heme oxygenase 1(HO-1) and NAD(P)H:quinine oxidoreductase 1 (NQO1)(Jeong, Jun and Kong., 2006, Zhao et al., 2014, Apopa, He and Ma., 2008). Our results demonstrated that LQHBG significantly alleviated stress-induced HREC damage via promoting Nrf2 and HO-1 activation (Figure6). The findings of the present study suggest that the protective ability of LQHBG involves activation of Nrf2/ARE/HO-1 signaling pathway and inhibition of NFkappa B signaling pathway.

LQHBG from Herba cistanches(RouCongRong), Radix Codonopsis(DanShen), Ganoderma lucidum (Ling Zhi), medlar(GouQiZi), Angelica sinensis(DangGui), Atractylodes chinensis(CangZhu), Chinese angelica(DangGui), etc., with Warming Yang exert tonifying effects on the kidney and invigorate the spleen to remove dampness to nourish the essence of the effect(Gong et al., 2008, Li et al., 2012).The main components of Cistanche glycosides reduce lipid peroxidation levels, effectively scavenge free radicals, and improve the respiratory function of mitochondria and oxidative DNA protection(Zhao et al., 2016, Deng et al., 2004, Li et al., 2016). Wolfberry reduces oxidative stress caused by free radical damage(Shi et al., 2018, Chang et al., 2018, Li et al., 2011). Codonopsis, angelica, chuanxiong, Ganoderma lucidum and other traditional Chinese medicines exhibit antioxidant effects(Gao et al., 2010, Saw et al., 2013, Chan et al., 2009, Zhu et al., 2015).

\section{Conclusions}

Previous studies have demonstrated that LQHB obviously protects the function and morphology of the retina from light-induced retinal damage in rats(Gong et al., 2008). LQHB has been used to treat retinal diseases in patients for over 17 years with great efficacy. Patient visual capacity is improved via the promotion of circulation in the choroids and retina, which prevents cell death, and reverses the inability to absorb and circulate vital nutrients for healthy vision. Conclusively, drug-containing LQHBG serum protects HRECs from oxidative stress-induced injury via reduced apoptosis and improved antioxidant 
capacity, which may involve activation of Nrf2/ARE/HO-1 signaling pathway and inhibition of NF-kappa B signaling pathway.

\section{Abbreviations}

LingQiHuangBan Granule LQHBG

Reactive oxygen stress ROS

Malondialdehyde MDA

Superoxide dismutase SOD

Catalase CAT

Glutathione peroxidase GSH-Px

Total antioxigenic capacity T-AOC

Poly ADP-ribose polymerase Parp

B-cell lymphoma-2 Bcl2

Tumor necrosis factor alpha TNFa

Human retinal endothelial cells HREC

Flow cytometry FCM

Central retinal artery occlusion CRAO

Central retinal vein occlusion CRVO

Branch retinal artery occlusion BRAO

Branch retinal vein occlusion BRVO

Age-related macular degeneration AMD

Terminaldeoxynucleotidyltransferase-mediated deoxyuridinetriphosphate-biotinnickendlabeling TUNEL Nuclear factor (erythroid-derived 2)-like $2 \mathrm{Nrf2}$

\section{Declarations}

Ethics approval and consent to participate 
Animals received humane care in accordance with the Association for Research in Vision and Ophthalmology(ARVO) Statement for the Use of Animals in Ophthalmic and Vision Research.

\section{Consent to publish}

No human was involved in this study.

\section{Availability of data and materials}

The raw data supporting the conclusions of this manuscript will be made available by the authors, without undue reservation, to any qualified researcher.

\section{Acknowledgments}

No

\section{Funding}

This study was supported by the Tibet Natural Science Foundation of China (No. XZ2017ZR-ZYZ09; to QHQ) and National Natural Science Foundation of China (No. 81300779; to ZZZ); National Key R\&D Program of China囚2016YFC0904800『2019YFC0840607; to QHQ and ZZ『and National Science and Technology Major Project of China $2017 Z X 09304010$,to QHQ and ZZ『in animal experiments, cell experiments, analysis, interpretation of data, and in writing the manuscript..

\section{Authors'contributions}

ZZZ and CDZ were involved in all aspects of research, analyzed data, wrote the paper, performed the experiments and analyzed data; QHQ and ZZ contributed to research design, supervision of research, data analysis and revised the manuscript; and TL and DD was involved in research design, supervision of the study, data analysis, and interpretation. All authors have read and approved the final manuscript.

\section{Competing interests}

The authors declare that there is no conflict of interest.

\section{References}

Agardh, C. D., C. Gustavsson, P. Hagert, M. Nilsson \& E. Agardh (2006) Expression of antioxidant enzymes in rat retinal ischemia followed by reperfusion. Metabolism, 55, 892-8.

Alfieri, A., S. Srivastava, R. C. Siow, M. Modo, P. A. Fraser \& G. E. Mann (2011) Targeting the Nrf2-Keap1 antioxidant defence pathway for neurovascular protection in stroke. J Physiol, 589, 4125-36.

Anderson, R. E., M. B. Maude \& J. C. Nielsen (1985) Effect of lipid peroxidation on rhodopsin regeneration. Curr Eye Res, 4, 65-71. 
Apopa, P. L., X. He \& Q. Ma (2008) Phosphorylation of Nrf2 in the transcription activation domain by casein kinase 2 (CK2) is critical for the nuclear translocation and transcription activation function of Nrf2 in IMR-32 neuroblastoma cells. J Biochem Mol Toxicol, 22, 63-76.

Atienzar-Aroca, S., M. Flores-Bellver, G. Serrano-Heras, N. Martinez-Gil, J. M. Barcia, S. Aparicio, D. PerezCremades, J. M. Garcia-Verdugo, M. Diaz-Llopis, F. J. Romero \& J. Sancho-Pelluz (2016) Oxidative stress in retinal pigment epithelium cells increases exosome secretion and promotes angiogenesis in endothelial cells. J Cell Mol Med, 20, 1457-66.

Bonne, C., A. Muller \& M. Villain (1998) Free radicals in retinal ischemia. Gen Pharmacol, 30, 275-80.

Castilho, A., C. A. Aveleira, E. C. Leal, N. F. Simoes, C. R. Fernandes, R. I. Meirinhos, F. I. Baptista \& A. F. Ambrosio (2012) Heme oxygenase-1 protects retinal endothelial cells against high glucose- and oxidative/nitrosative stress-induced toxicity. PLoS One, 7, e42428.

Chan, J. Y., F. C. Lam, P. C. Leung, C. T. Che \& K. P. Fung (2009) Antihyperglycemic and antioxidative effects of a herbal formulation of Radix Astragali, Radix Codonopsis and Cortex Lycii in a mouse model of type 2 diabetes mellitus. Phytother Res, 23, 658-65.

Chang, J. S., Y. J. Lee, D. A. Wilkie \& C. T. Lin (2018) The Neuroprotective and antioxidative effects of submicron and blended Lycium barbarum in experimental retinal degeneration in rats. $J$ Vet Med Sci, 80, 1108-1115.

Chen, B., S. Caballero, S. Seo, M. B. Grant \& A. S. Lewin (2009) Delivery of antioxidant enzyme genes to protect against ischemia/reperfusion-induced injury to retinal microvasculature. Invest Ophthalmol Vis Sci, 50, 5587-95.

Das \& H. Yu (2012) HINT: High-quality protein interactomes and their applications in understanding human disease. BMC Systems Biology, 30;6(1):92.

de Vries, H. E., M. Witte, D. Hondius, A. J. Rozemuller, B. Drukarch, J. Hoozemans \& J. van Horssen (2008) Nrf2-induced antioxidant protection: a promising target to counteract ROS-mediated damage in neurodegenerative disease? Free Radic Biol Med, 45, 1375-83.

Deliyanti, D., S. F. Alrashdi, S. M. Tan, C. Meyer, K. W. Ward, J. B. de Haan \& J. L. Wilkinson-Berka (2018) Nrf2 Activation Is a Potential Therapeutic Approach to Attenuate Diabetic Retinopathy. Invest Ophthalmol Vis Sci, 59, 815-825.

Deng, M., J. Y. Zhao, X. D. Ju, P. F. Tu, Y. Jiang \& Z. B. Li (2004) Protective effect of tubuloside B on TNFalpha-induced apoptosis in neuronal cells. Acta Pharmacol Sin, 25, 1276-84.

Di Mascio, P., M. E. Murphy \& H. Sies (1991) Antioxidant defense systems: the role of carotenoids, tocopherols, and thiols. Am J Clin Nutr, 53, 194S-200S. 
Enomoto, A., K. Itoh, E. Nagayoshi, J. Haruta, T. Kimura, T. O'Connor, T. Harada \& M. Yamamoto (2001) High sensitivity of Nrf2 knockout mice to acetaminophen hepatotoxicity associated with decreased expression of ARE-regulated drug metabolizing enzymes and antioxidant genes. Toxicol Sci, 59, 169-77.

Erdelyi, K., E. Bakondi, P. Gergely, C. Szabo \& L. Virag (2005) Pathophysiologic role of oxidative stressinduced poly(ADP-ribose) polymerase- 1 activation: focus on cell death and transcriptional regulation. Cell Mol Life Sci, 62, 751-9.

Gao, Y., X. G. Deng, Q. N. Sun \& Z. Q. Zhong (2010) Ganoderma spore lipid inhibits N-methyl-Nnitrosourea-induced retinal photoreceptor apoptosis in vivo. Exp Eye Res, 90, 397-404.

Gong, Y. Y., Y. Song, Z. G. Xie \& X. W. Wu (2008) Protective effect of Huangban Granule against lightinduced retinal damage in rats. Zhong Xi Yi Jie He Xue Bao, 6, 1159-63.

Huang, Da.,W. Sherman \&R.,A, Lempicki(2009) Bioinformatics enrichment tools: paths toward the comprehensive functional analysis of large gene lists[J]. Nucleic Acids Res, 2009, 37(1): 1-13.

Jeong, W. S., M. Jun \& A. N. Kong (2006) Nrf2: a potential molecular target for cancer chemoprevention by natural compounds. Antioxid Redox Signal, 8, 99-106.

Leal, E. C., C. A. Aveleira, A. F. Castilho, A. M. Serra, F. I. Baptista, K. Hosoya, J. V. Forrester \& A. F. Ambrosio (2009) High glucose and oxidative/nitrosative stress conditions induce apoptosis in retinal endothelial cells by a caspase-independent pathway. Exp Eye Res, 88, 983-91.

Levin, L. A. \& A. Louhab (1996) Apoptosis of retinal ganglion cells in anterior ischemic optic neuropathy. Arch Ophthalmol, 114, 488-91.

Lewden, O., C. Garcher, C. Morales, A. Javouhey, L. Rochette \& A. M. Bron (1996) Changes of catalase activity after ischemia-reperfusion in rat retina. Ophthalmic Res, 28, 331-5.

Li, C. H., Q. H. Qiu, X. W. Wu, Y. Y. Gong, Z. G. Xie, Y. Song \& Q. Gu (2012) [Chinese herbal medicine Lingqi Huangban Granule protects retinal pigment epithelial cells against oxidative stress-induced injury in vitro]. Zhong Xi Yi Jie He Xue Bao, 10, 85-90.

Li, S. Y., D. Yang, C. M. Yeung, W. Y. Yu, R. C. Chang, K. F. So, D. Wong \& A. C. Lo (2011) Lycium barbarum polysaccharides reduce neuronal damage, blood-retinal barrier disruption and oxidative stress in retinal ischemia/reperfusion injury. PLoS One, 6, e16380.

Li, Z., H. Lin, L. Gu, J. Gao \& C. M. Tzeng (2016) Herba Cistanche (Rou Cong-Rong): One of the Best Pharmaceutical Gifts of Traditional Chinese Medicine. Front Pharmacol, 7, 41.

Lu, W. X. W, Zhang. L(2016)Clinical Study of "Lingqi Huangban Granule" in Treating Choroidal Neovascularisation in Pathologic Myopia. Chinese Medicine, 7, 125-132. 
Masuda, T., M. Shimazawa \& H. Hara (2017) Retinal Diseases Associated with Oxidative Stress and the Effects of a Free Radical Scavenger (Edaravone). Oxid Med Cell Longev, 2017, 9208489.

Osborne, N. N., R. J. Casson, J. P. Wood, G. Chidlow, M. Graham \& J. Melena (2004) Retinal ischemia: mechanisms of damage and potential therapeutic strategies. Prog Retin Eye Res, 23, 91-147.

Pellegrini-Giampietro, D. E., G. Cherici, M. Alesiani, V. Carla \& F. Moroni (1990) Excitatory amino acid release and free radical formation may cooperate in the genesis of ischemia-induced neuronal damage. $J$ Neurosci, 10, 1035-41.

Poornima, P., Kumar, J. D., Zhao, Q., Blunder, M., and Efferth, T. (2016). Network pharmacology of cancer: from understanding of complex interactomes to the design of multi-target specific therapeutics from nature. Pharmacol. Res. 111, 290-302.

Saw, C. L., Q. Wu, Z. Y. Su, H. Wang, Y. Yang, X. Xu, Y. Huang, T. O. Khor \& A. N. Kong (2013) Effects of natural phytochemicals in Angelica sinensis (Danggui) on Nrf2-mediated gene expression of phase II drug metabolizing enzymes and anti-inflammation. Biopharm Drug Dispos, 34, 303-11.

Shannon, P. Markiel \& O. Ozier (2003)Cytoscape: a software environment for integrated models of biomolecular interaction networks[J]. Genome Res, 13(11): 2498-504.

Shi, G. J., J. Zheng, X., X. Han, Y., P. Jiang, Z.,M. Li, J. Wu, Q. Chang, Y. Niu, T. Sun, Y. X. Li, Z. Chen \& J. Q. $\mathrm{Yu}$ (2018) Lycium barbarum polysaccharide attenuates diabetic testicular dysfunction via inhibition of the PI3K/Akt pathway-mediated abnormal autophagy in male mice. Cell Tissue Res.

Shi, K., X. Wang, J. Zhu, G. Cao, K. Zhang \& Z. Su (2015) Salidroside protects retinal endothelial cells against hydrogen peroxide-induced injury via modulating oxidative status and apoptosis. Biosci Biotechnol Biochem, 79, 1406-13.

Tang, J., and Aittokallio, T. (2014). Network pharmacology strategies toward multitarget anticancer. therapies: from computational models to experimental design principles. Curr. Pharm. Des. 20, 23-36.

Zhang, L., L. Dong, X. Liu, Y. Jiang, L. Zhang, X. Zhang, X. Li \& Y. Zhang (2014) alpha-Melanocytestimulating hormone protects retinal vascular endothelial cells from oxidative stress and apoptosis in a rat model of diabetes. PLoS One, 9, e93433.

Zhang, Y., C. H. Cho, L. O. Atchaneeyasakul, T. McFarland, B. Appukuttan \& J. T. Stout (2005) Activation of the mitochondrial apoptotic pathway in a rat model of central retinal artery occlusion. Invest Ophthalmol Vis Sci, 46, 2133-9.

Zhao, J., B. Zhang, S. Li, L. Zeng, Y. Chen \& J. Fang (2014) Mangiferin increases Nrf2 protein stability by inhibiting its ubiquitination and degradation in human HL60 myeloid leukemia cells. Int J Mol Med, 33, 1348-54. 
Zhao, Q., X. Yang, D. Cai, L. Ye, Y. Hou, L. Zhang, J. Cheng, Y. Shen, K. Wang \& Y. Bai (2016) Echinacoside Protects Against MPP(+)-Induced Neuronal Apoptosis via ROS/ATF3/CHOP Pathway Regulation.

Neurosci Bull, 32, 349-62.

Zhu, X., K. Wang, K. Zhang, X. Tan, Z. Wu, S. Sun, F. Zhou \& L. Zhu (2015) Tetramethylpyrazine Protects Retinal Capillary Endothelial Cells (TR-iBRB2) against IL-1beta-Induced Nitrative/Oxidative Stress. Int J Mol Sci, 16, 21775-90.

Xu, H., Y. Zhang, Y.Q, Liu, Z.,M. Chen, T. Lv, C.,Y. Tang, S.,H. Zhang, X.,B. Zhang, W. Li, Z.,Y. Zhou, R.,R. Yang, H.,J. Wang \& L.Q. Huang(2018) ETCM: an encyclopaedia of traditional Chinese medicine. Nucleic Acids Res, 26. doi: 10.1093/nar/gky987.

\section{Figures}

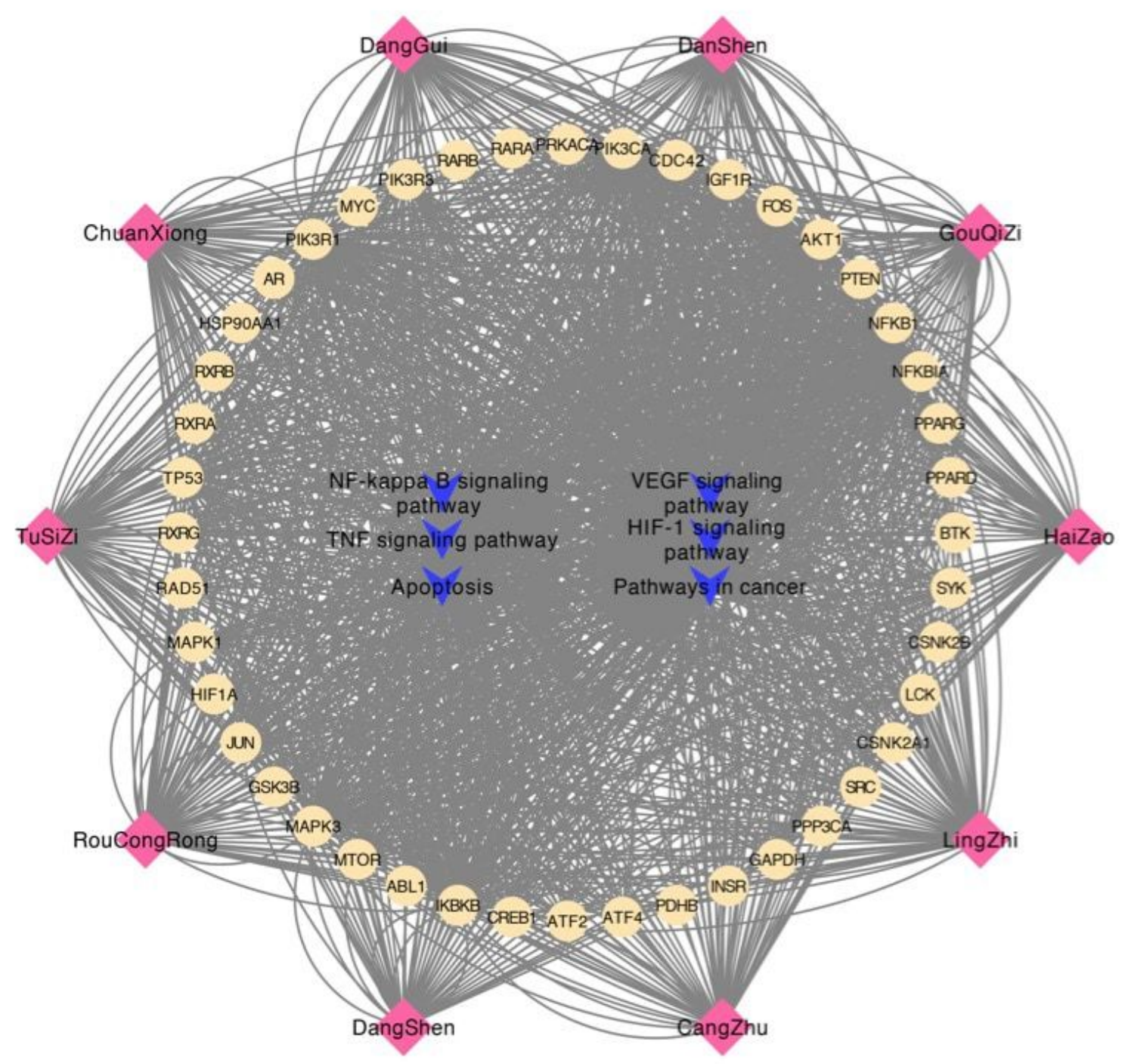

Figure 1 
Signaling pathways for network construction.

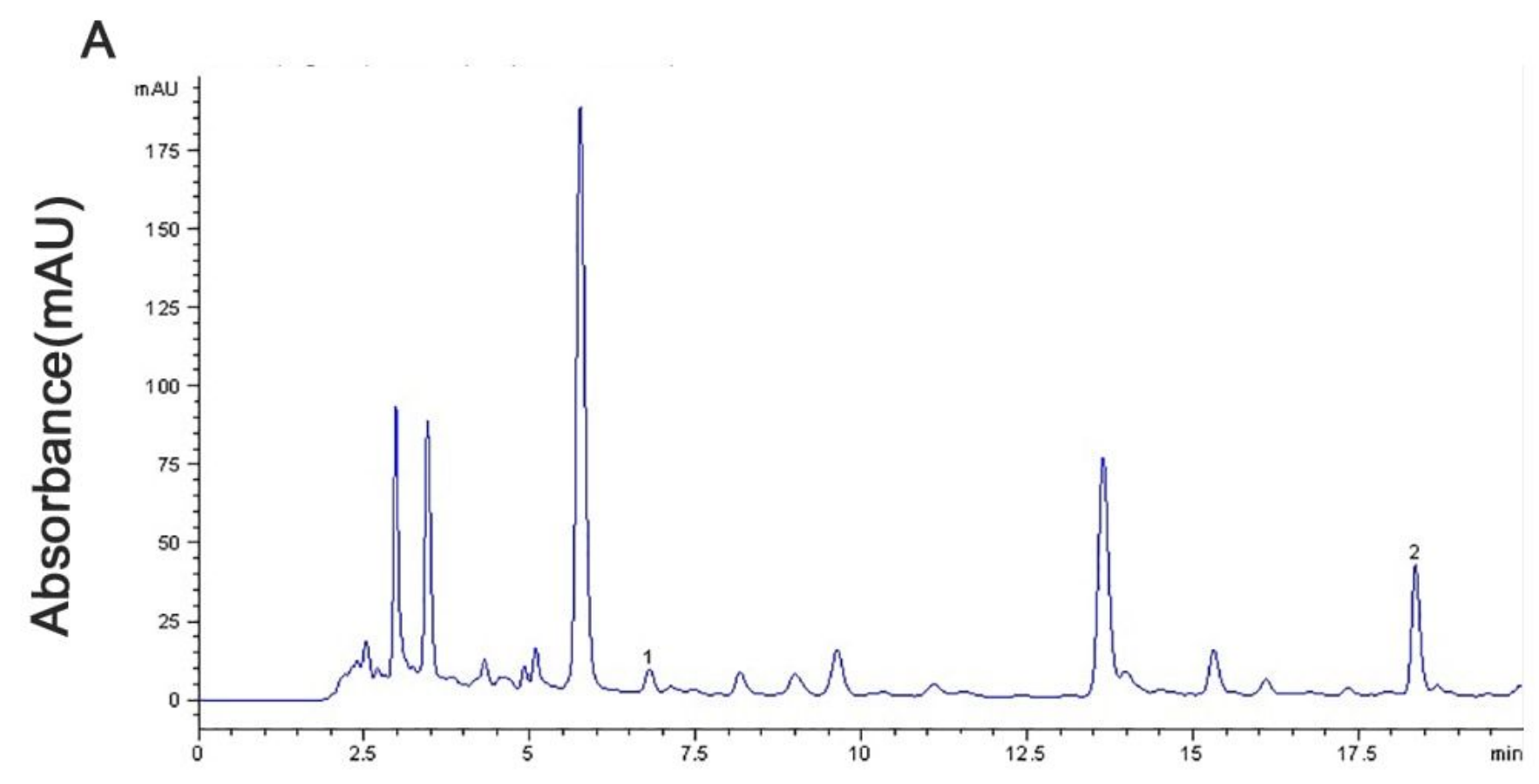

B

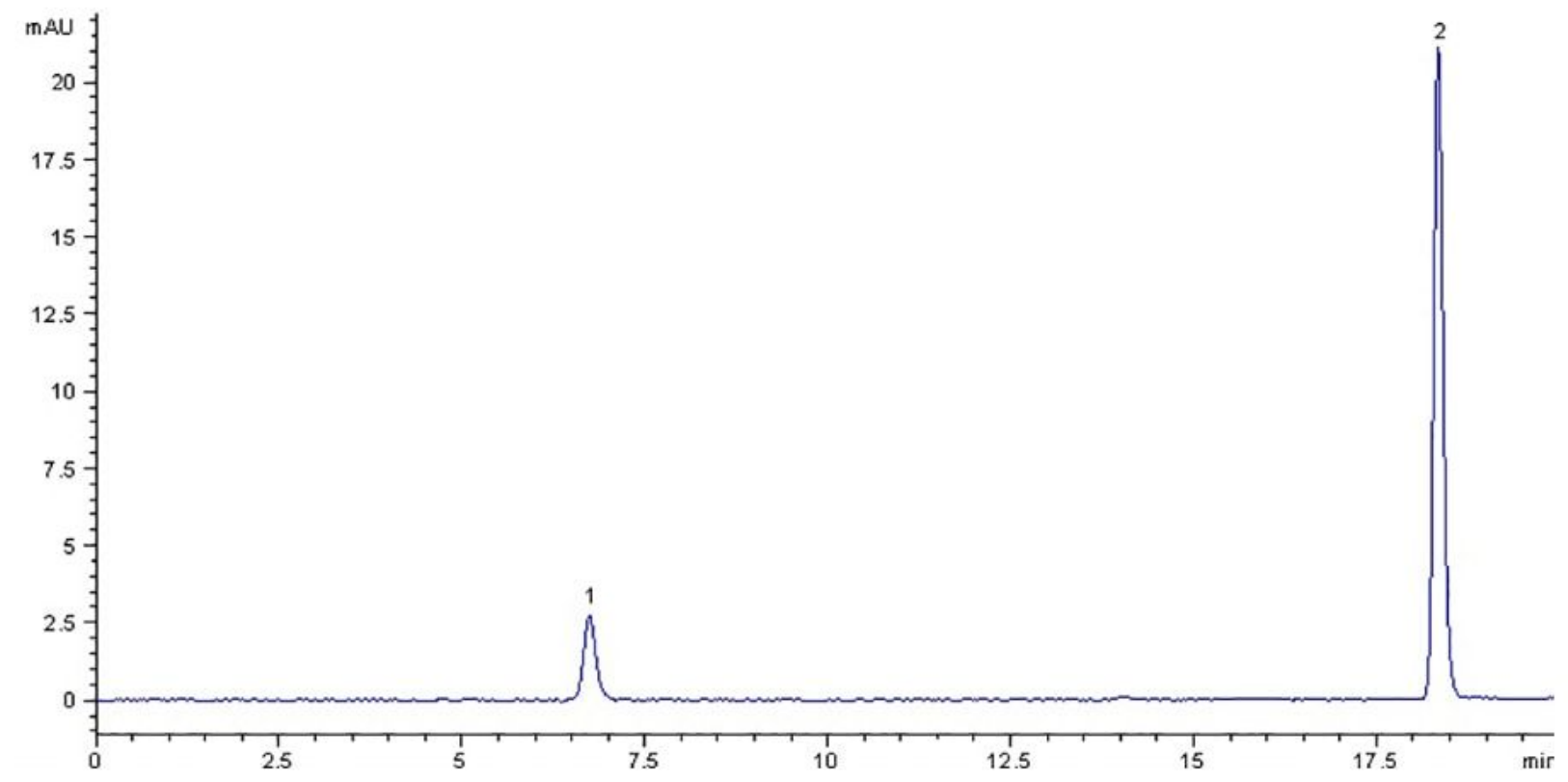

Figure 2

Chromatogram of LQHBG and standard chemicals mixture analyzed by HPLC.1. Acteoside ; 2 . salvianolic acid. 

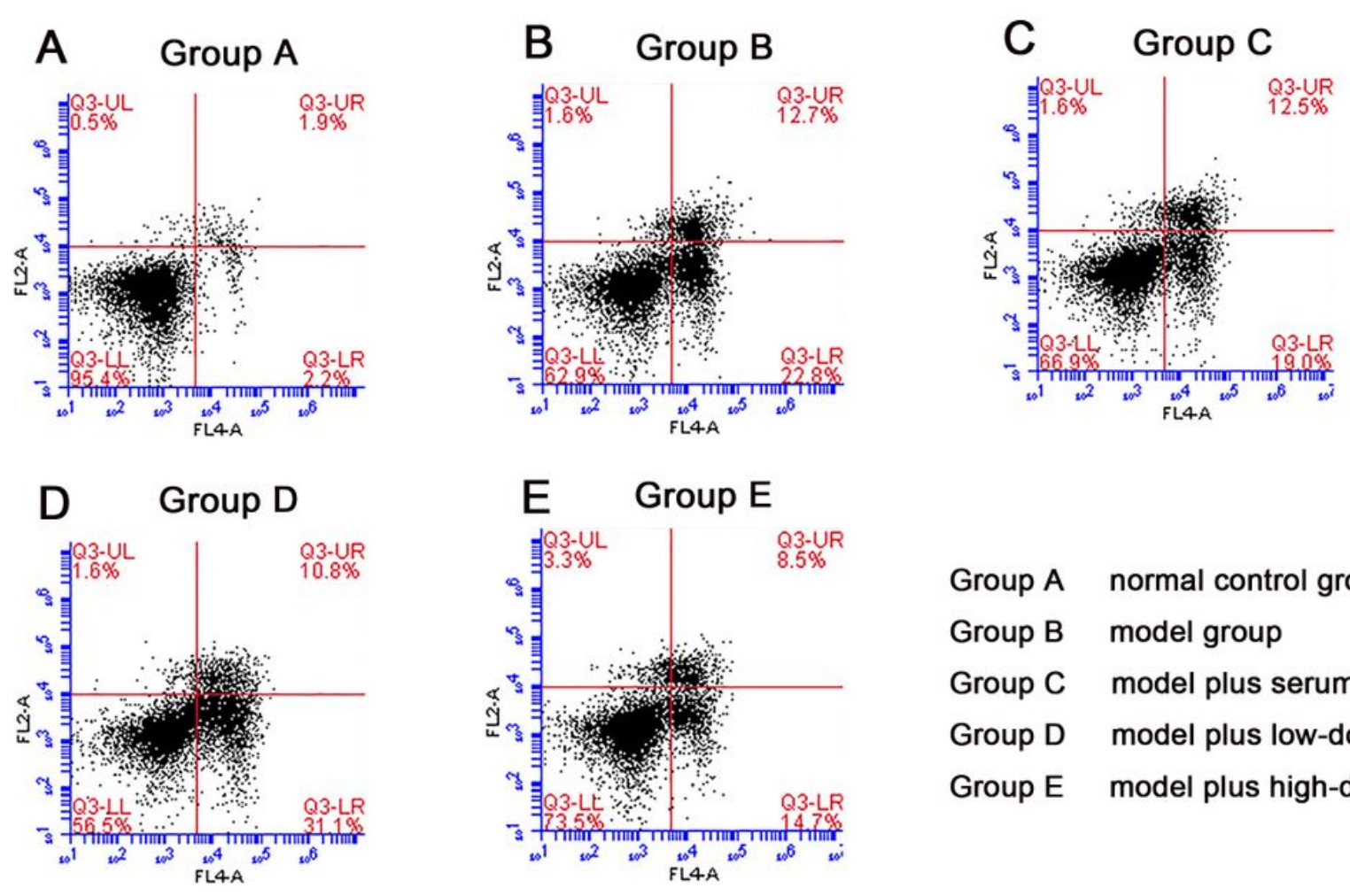
Group A normal control group
Group B model group
Group C model plus serum goup
Group D model plus low-dose LQHBG group
Group E model plus high-dose LQHBG group

$\mathrm{F}$

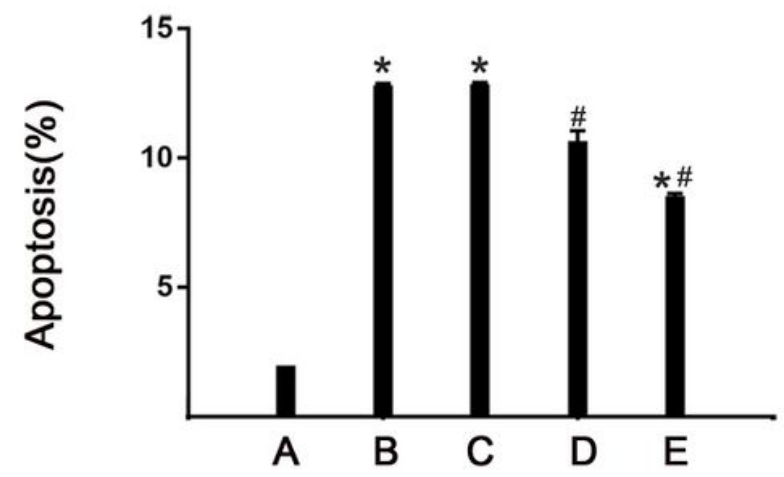

\section{Figure 3}

Detection of cell apoptosis via flow cytometer. (A) Normal control group with the fewest apoptotic cells apoptosis. $(B, C)$ Model group and blank control group, the levels of cell apoptosis was similar, the difference between two groups was not significant $(P>0.05)$. $\triangle \mathrm{D}, \mathrm{E} \otimes T$ The apoptosis levels of low dose and high dose drug plus model group was significantly reduced, with statistically significant difference $(\mathrm{P}<$ 0.01) compared with model group. (F) The difference of apoptosis level between the high dose drug plus model group and low dose drug plus model group has statistical significance $(P<0.05)$. 

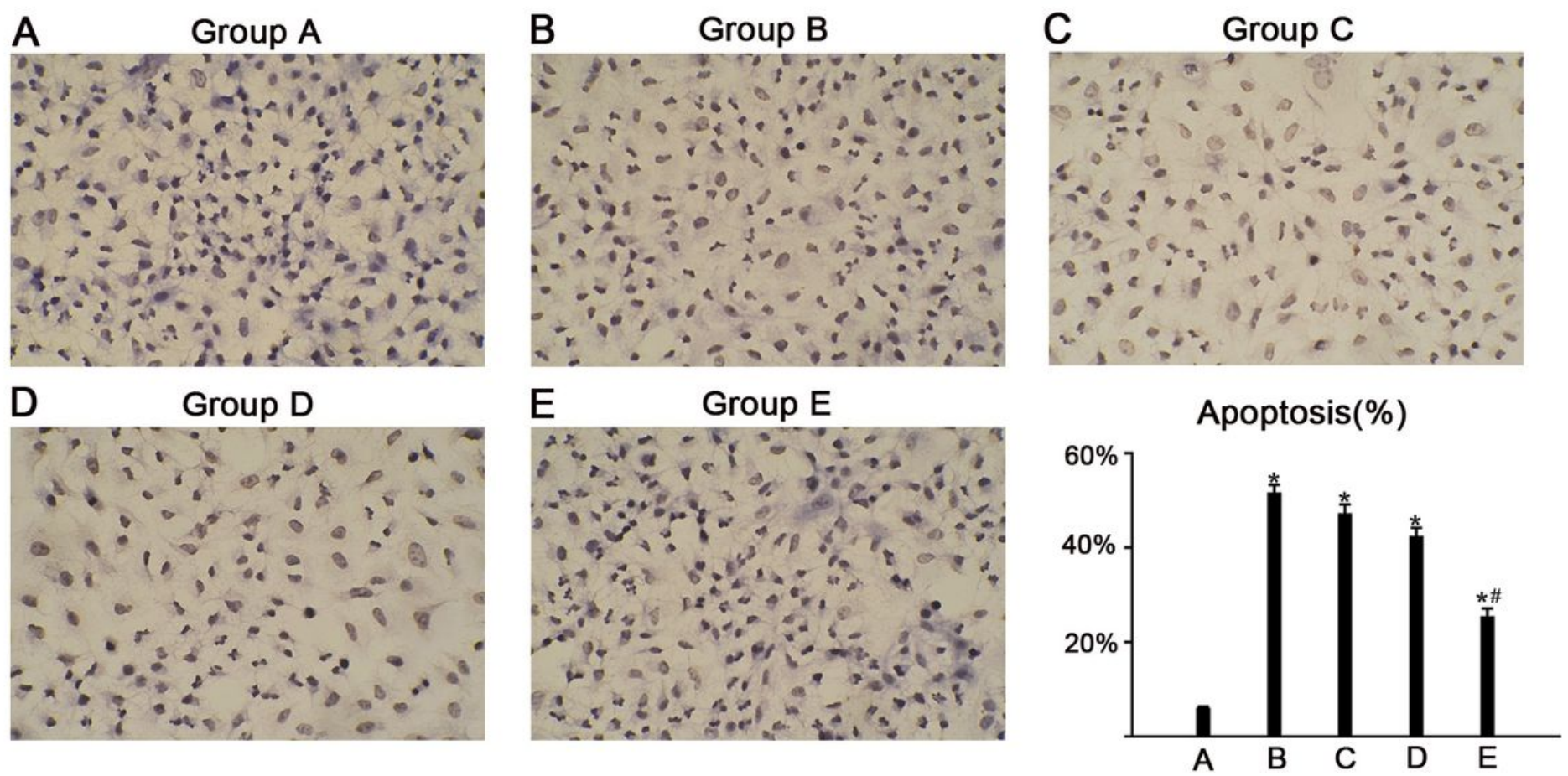

Group A normal control group

Group B model group

Group C model plus serum goup

Group D model plus low-dose LQHBG group

Group E model plus high-dose LQHBG group

\section{Figure 4}

TUNEL detection of HREC cell apoptosis. (A) Normal control group, TUNEL staining was negative, no yellow HRECs nuclei. $(B, C)$ Model group and blank serum group visible large yellow TUNEL positive staining in the HRECs nuclei. (D) Low dose drug plus model group with a small, scattered yellow TUNEL positive staining of HRECs nuclei. (E) In high dose drug plus model group, yellow TUNEL positive staining in the nuclei of HRECs is further reduced, significantly less than the number of model group, the difference between low dose and high dose drug plus model groups has statistical significance $(\mathrm{P}<$ $0.05)$. 

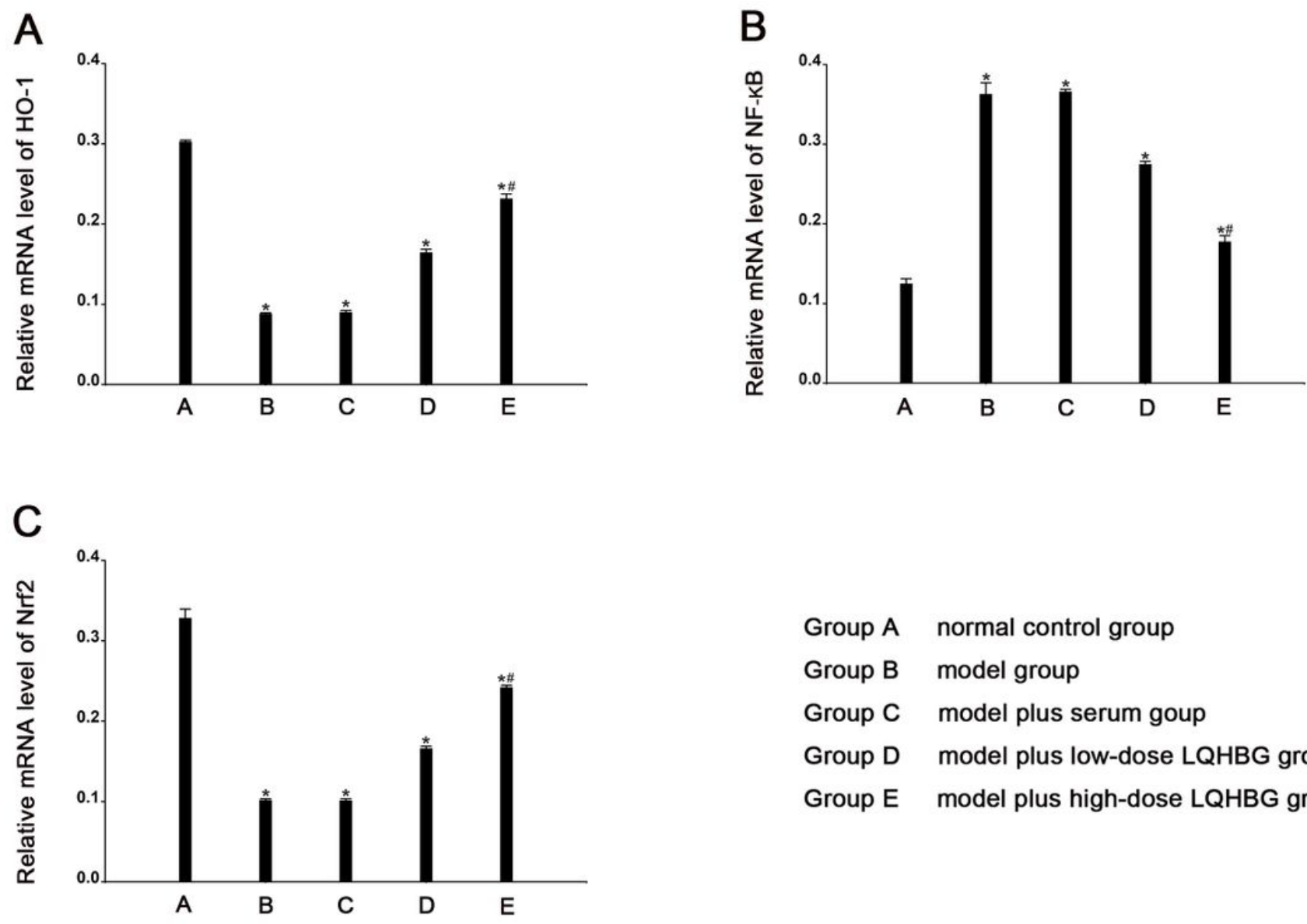
Group A normal control group
Group B model group
Group C model plus serum goup
Group D model plus low-dose LQHBG group
Group E model plus high-dose LQHBG group

\section{Figure 5}

LQHBG treatment reduced activation of NF-KB, and attenuated the downregulation of HO-1, Nrf2mRNA in HRECs. (B): RT-PCR analysis shows significant activation of NF-KB in model group and blank serum group; LQHBG dose-dependently reduced the activation. Histograms represent the mean+SEM of NF-KB mRNA levels in all groups.*: $p<0.01$ versus normal control group; \#: $p<0.01$ versus model group. $(A, C)$ : Histograms represent the mean+SEM of HO-1, Nrf2 mRNA levels in model and blank serum group were strongly reduced compared with normal control group; LQHBG dose-dependently attenuated the reduction of HO-1, Nrf2 expression. Histograms represent the mean+SEM of HO-1, Nrf2mRNA levels in all groups.*: $p<0.01$ versus normal control group; \#: $p<0.01$ versus model group. 

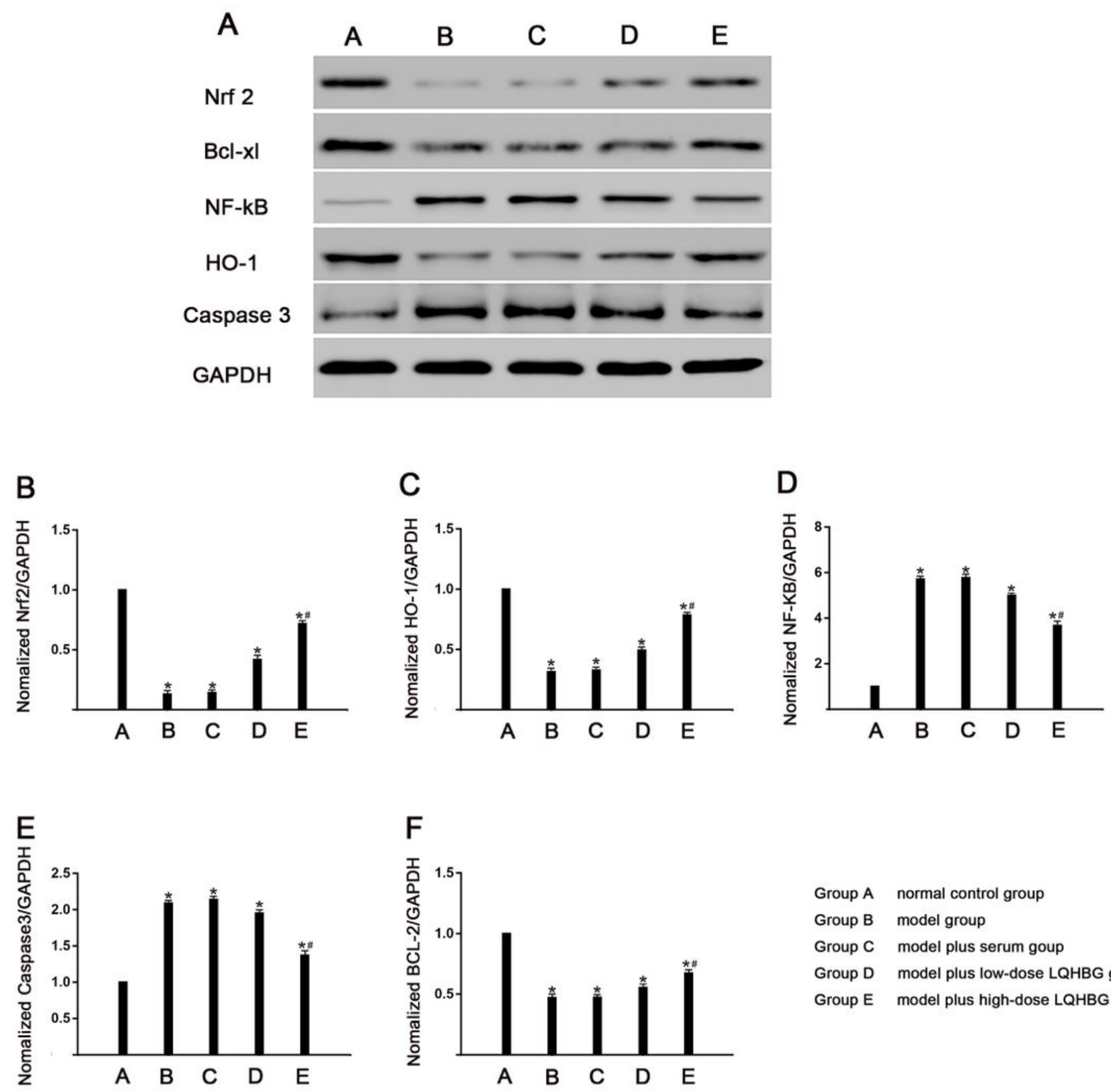
Group A normal control group
Group B model group
Group C model plus serum goup
Group D model plus low-dose LQHBG group
Group E model plus high-dose LQHBG group

Figure 6

LQHBG reduced release of caspase3, NF-KB, and attenuated the reduction of $\mathrm{Nrf} 2, \mathrm{HO}-1, \mathrm{Bcl}-2$ protein expressions in HRECs. (A,D,E): Western-blot analysis shows significant activation of caspase3, NF-KB in model group and blank serum model group; LQHBG treatment dose-dependently reduced these effects*: $p<0.01$ versus normal control group ; $\#$ : $p<0.01$ versus model group. $(A, B, C, F)$ : Histograms represent the mean+SEM of Nrf2, HO-1, Bcl-2 protein levels in retinas of all groups. The HO-1, Nrf2, Bcl-2 protein levels in model and blank serum group were strongly reduced compared with normal control group; LQHBG dose-dependently attenuated the reduction of $\mathrm{HO}-1, \mathrm{Nrf} 2, \mathrm{Bcl}-2$ protein expression. Histograms represent 
the mean+SEM of HO-1, Nrf2, Bcl-2 protein levels in all groups.*: $p<0.01$ versus normal control group; \#: $p<0.01$ versus model group.*: $p<0.01$ versus normal control group; $\#$ : $p<0.01$ versus model group.

A

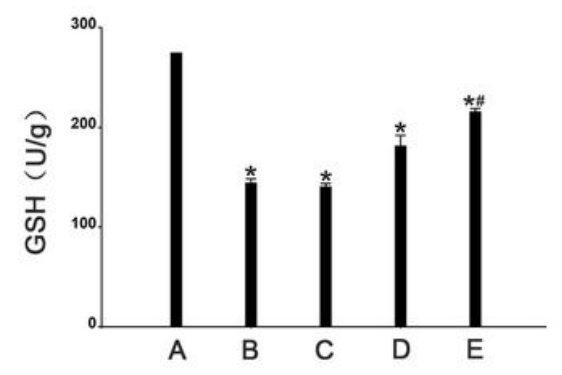

D

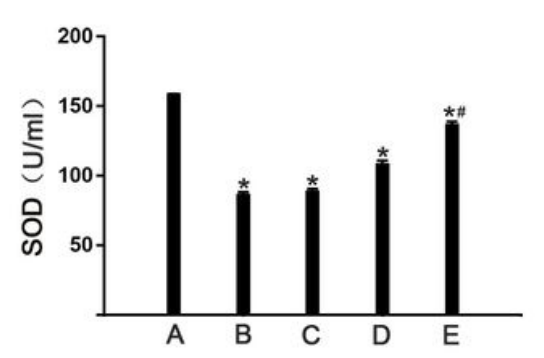

B

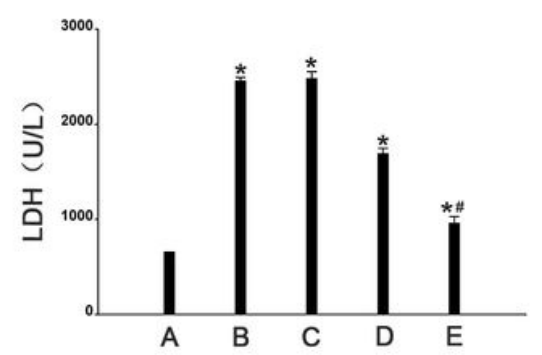

$E$

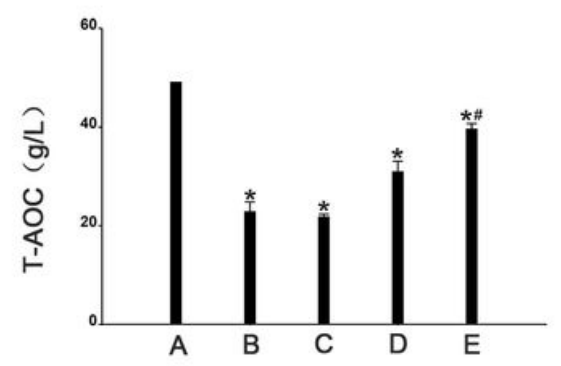

C

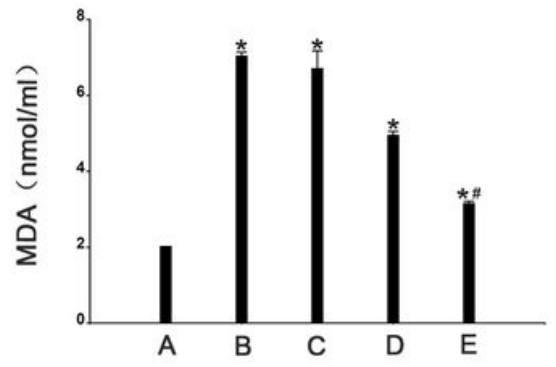

Group A normal control group

Group B model group

Group C model plus serum goup

Group D model plus low-dose LQHBG group

Group E model plus high-dose LQHBG group

Figure 7

Changes in MDA levels and SOD, GSH-Px, LDH, T-AOC activities in HRECs. (A): MDA, LDH levels in model group and blank serum model group became significantly higher than normal control group $(p<0.05)$; MDA, LDH levels in low dose and high dose drug plus model groups were higher than normal control group, but were significantly lower than model group $(p<0.05)$. LQHBG dose-dependently attenuated the activation of MDA, LDH levels.*: $p<0.05$ versus normal control group; $\#$ : $p<0.05$ versus model group. (B, $C$, D): Activities of SOD, GSH-Px, and T-AOC in model group and model plus serum group became significantly lower than those of normal control group $(p<0.05)$; Activities in low dose and high dose drug plus model groups were significantly higher than those of model group $(p<0.05)$. LQHBG dosedependently attenuated the reduction of SOD, GSH-Px and T-AOC levels.*: $p<0.05$ versus normal control group; \#: $p<0.05$ versus model group.

\section{Supplementary Files}

This is a list of supplementary files associated with this preprint. Click to download.

- TableS1.xIsx

- Tables3.xIsx

- Tables2.xIsx 